The 13th European Nutrition Conference, FENS 2019, was held at the Dublin Convention Centre, 15-18 October 2019

\title{
The phenomenon of zinc deficiency among children in China aged 3-5 years old
}

\section{Abstract}

Introduction: zinc is an essential micro-nutrient for growth and proper immune function. Yet there are limited data available on the prevalence of zinc deficiency among children aged 3-5 at the country level. This information will enable health planners to determine the need for zinc intervention activities and to stimulate further research into these areas.

materials and methods: The data on children aged 3-5 were extracted from the Chinese National Nutrition and Health Surveillance in 2013. By multi-stage stratified cluster randomly sampling method, 30 children aged 3-5 years old were selected from each region for this study from 55 counties in China to analyze serum zinc. Finally, 1472 children aged 3-5 years were included in the study. The concentration of serum zinc was determined by high resolution inductively coupled plasm mass spectrometry. High and low level quality control samples were used, measured value was $(1.63 \pm 0.04) \mathrm{mg} / \mathrm{l}$ and $(2.80 \pm 0.06) \mathrm{mg} / \mathrm{l}$, respectively. CV of quality control samples were $1.69 \% \sim 2.45 \%$. The zinc deficiency was defined as serum zinc level $<70 \mu \mathrm{g} / \mathrm{dl}$ with the standard of WHO.

Results: serum zinc means of children aged $3-5$ years was $(95.3 \pm 18.2) \mu \mathrm{g} / \mathrm{dl}$ and $3.9 \%$ children with zinc deficiency. serum zinc means level in urban children was $(98.9 \pm 17.6) \mu \mathrm{g} / \mathrm{dl}$, and $(91.6 \pm 18.2) \mu \mathrm{g} / \mathrm{dl}$ in rural area. we showed that the serum zinc deficiency rate was higher in rural children $(5.5 \%)$ than urban children $(2.4 \%)$, and there were significant differences between these two areas. serum zinc means level in boys aged 3-5 years was $(95.3 \pm 18.7) \mu \mathrm{g} / \mathrm{dl}$, and $(95.3 \pm 17.8) \mu \mathrm{g} / \mathrm{dl}$ in girls aged $3-5$ years old. The prevalence of zinc deficiency was $1.5 \%, 6.6 \%$ and $1.8 \%$ in $3 \sim, 4 \sim, 5 \sim$ years old urban boys, respectively; $6.8 \%, 7.7 \%$ and $4.0 \%$ in rural boys, respectively. The prevalence of zinc deficiency was $2.3 \%, 0.8 \%$ and $1.7 \%$ in $3 \sim, 4 \sim, 5 \sim$ years old urban girls, respectively; $4.1 \%, 7.0 \%$ and $4.0 \%$ in rural girls, respectively. And there were differences between urban and rural areas in girls of $4 \sim .5$ years.

Discussion: The zinc level of children aged 3-5 years in China has been improved compared with ten years ago, but the zinc deficiency of rural children is still lower than that of urban children, especially those aged 4 to 5 years in rural areas, so we should pay more attention to this group.

\section{Conflict of Interest}

I don't have any commercial interests or associations that might pose a conflict of interest regarding this submission 